\title{
Chronic neutropenia following gold therapy
}

\author{
DAVID A. SISAM, DO \\ JOHN P. SHEEHAN, MD
}

We describe the first case of chronic neutropenia of 17 years' duration following gold therapy in a 53-year-old woman given a 1-g course of gold therapy in $\mathbf{1 9 6 5}$ for treatment of seropositive rheumatoid arthritis. Although she had a good response to the gold therapy, her originally normal leukocyte count fell to $1.2 \times$ $10^{9} / \mathrm{L}$. Over the subsequent 17 years, she required multiple hospitalizations for recurrent skin, mouth, and respiratory tract infections.

Serial leukocyte counts failed to show a cyclical nature to the chronic neutropenia. Normal results of a technetium Tc 99m spleen scan and lack of increased bone marrow leukocyte precursors rendered a diagnosis of Felty's syndrome unlikely. A bone marrow biopsy specimen revealed an isolated reduction in the number of myeloid precursors, which is consistent with gold-induced bone marrow toxicity. This patient's relative freedom from serious life-threatening infections remains enigmatic, but is undoubtedly related to her ability to augment another phagocytic cell line, and the remarkable phagocytic activity of her monocytes appears to have well compensated for her neutropenia.

Dr Sisam is director of the Regional Diabetes Management Center, Amherst, Ohio, and Dr Sheehan is assistant professor of medicine, Case Western Reserve University, Cleveland.

Reprint requests to John P. Sheehan, MD, 2074 Abington, Rm 3124, Cleveland, OH 44106.
This activity was most likely responsible for her long-term survival.

Neutropenia is a well-recognized hazard of gold therapy, and generally is a transient phenomenon with either complete resolution or fatality from overwhelming sepsis. We describe the first case, to our knowledge, of chronic neutropenia of 17 years' duration following gold therapy.

\section{Report of a case}

A 53-year-old woman, who fulfilled the American Rheumatism Association's criteria for rheumatoid arthritis, was given a 1-g course of gold therapy in 1965 for treatment of seropositive rheumatoid arthritis. Toward the end of the course of gold therapy, she was found to have developed acute neutropenia (Fig 1). She was not receiving other medications at that time. She responded well to the gold therapy from the standpoint of her arthritic condition. Following cessation of gold therapy, she required only nonsteroidal anti-inflammatory agents for treatment of mild symptoms. In the following 13 years, the patient had recurrent skin infections following minor trauma, recurrent mouth infections, and, on an average, four respiratory tract infections per year. In 1978, she was hospitalized with progressive dysphagia of 4 months' duration, general malaise, and weight loss. Results of a physical examination at that time were unremarkable, with no splenomegaly evident, no rheumatoid nodules, and no deformities.

Her leukocyte count was $1.1 \times 10^{9} / \mathrm{L}$, with a differential cell count of 0.30 polymorphonuclear leukocytes, 0.36 lymphocytes, 0.32 monocytes, and 0.02 basophils. The erythrocyte count was $4.85 \times$ 


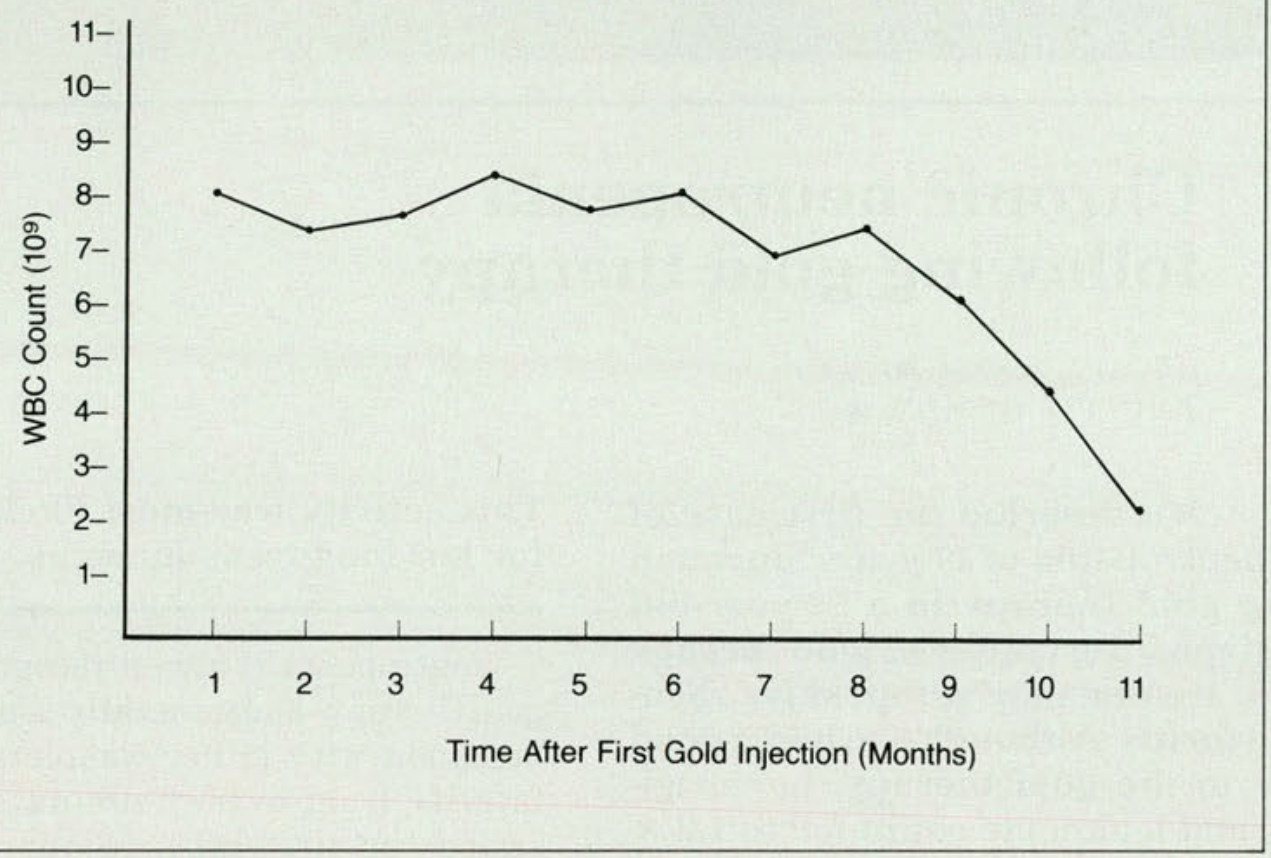

Figure 1. Serial WBC counts at monthly intervals while patient was receiving gold injections show development of acute neutropenia toward end of 1-g course.

$10^{12} / \mathrm{L}$, the hemoglobin level was $11.1 \mathrm{~g} / \mathrm{dL}$, the hematocrit was 0.34 , and the mean corpuscular volume was $70 \mathrm{fL}$. The platelet count was $345 \times 10^{9 /}$ L. The erythrocyte sedimentation rate was $84 \mathrm{~mm} /$ h. A bone marrow biopsy revealed a reduction in myeloid precursors with normal megakaryocytes and normal erythrocyte precursors. Rheumatoid factor was positive at $1: 1600$. Results of a sheep cell agglutination test were positive at $1: 64$, antinuclear antibodies were present at a titer of $1: 80$, and anti-DNA antibody titer was positive (Farr, $30 \%$ ). Immunoglobulin levels were normal. Antigranulocyte antibodies were not measured (not routinely available in 1980 ).

Results of a technetium Tc $99 \mathrm{~m}$ sulfur colloid scan revealed a normal size spleen. Results of a barium swallow revealed a stricture at the lower end of the esophagus, with mucosal ulceration and free esophageal reflux. Esophagoscopy confirmed a peptic stricture at $30 \mathrm{~cm}$; the stricture was easily dilated.

In the next 4 years, repeated esophagoscopy and successful dilatation were carried out on six occasions. During this time, the patient suffered four episodes of pneumonia requiring hospitalization. Some of these occurred in "dependent" locations (aspiration); others did not. Mouth infections tended to be troublesome, and Streptococcus species were generally isolated on culture.

Results of serial leukocyte counts over a 2-month period failed to reveal a cyclical nature to the chronic neutropenia (see Fig 2). Results of the differential cell counts during periods of infection showed a pronounced reactive monocytosis, with the monocyte counts being in excess of 0.80 on occasion. There was never any clinical evidence of other causes of monocytosis (monocytic leukemia, toxoplasmosis, mononucleosis, cytomegalovirus). Results of monocyte function studies confirmed a remarkable phagocytic activity of the patient's monocytes in vitro.

\section{Comment}

Bone marrow toxicity is a well-recognized hazard of gold therapy, and the bone marrow findings in the current case are similar to those documented in acute gold-induced neutropenia. ${ }^{1}$ Susceptibility to gold-induced neutropenia is thought possibly to be an individual idiosyncratic response, perhaps related to altered metabolism, resulting in high local con- 


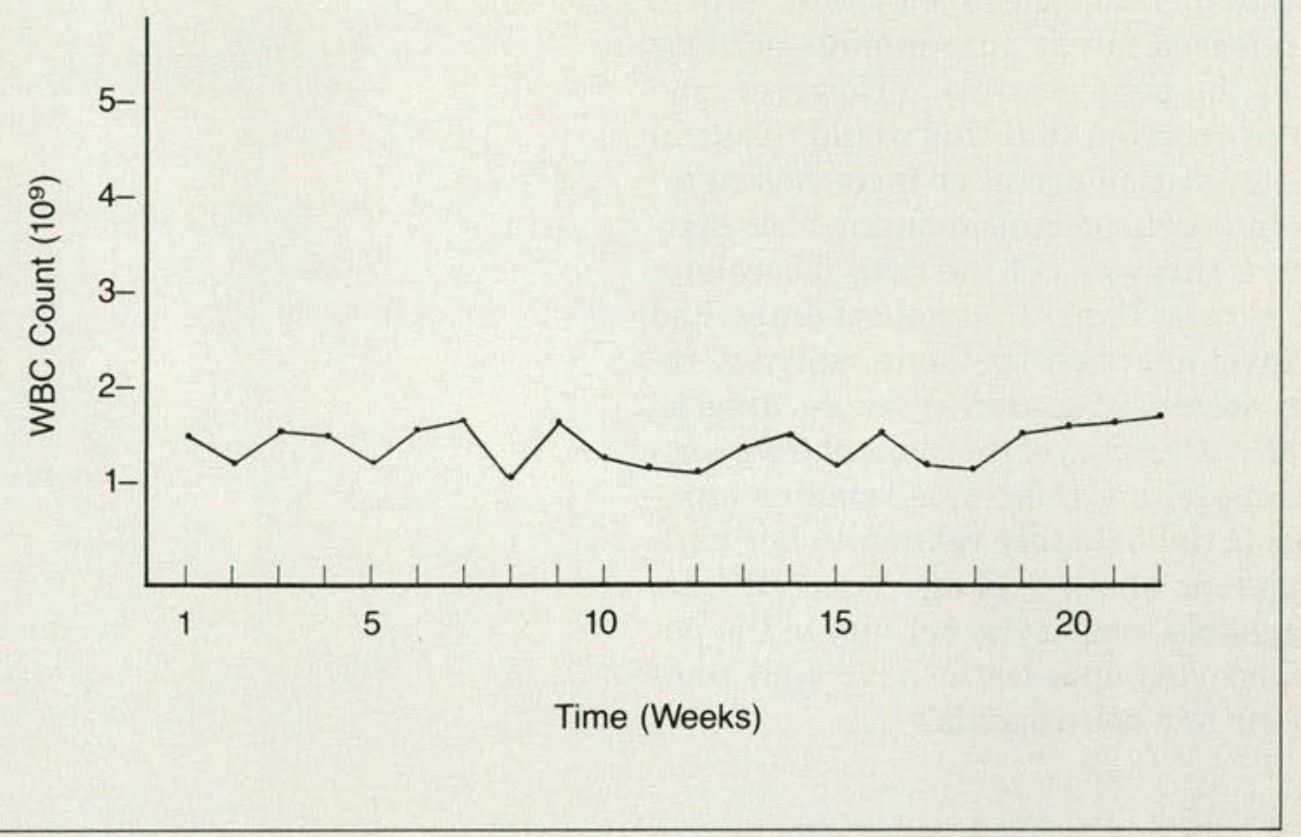

Figure 2. Serial WBC counts at weekly intervals over a 6 -month period after the course of gold therapy fail to reveal any cyclical nature to patient's chronic netropenia.

centrations of free gold in the bone marrow, ${ }^{2}$ specific biochemical defects such as decreased pyrimidine biosynthesis, ${ }^{3}$ or immunologic mechanisms. ${ }^{4}$ Although gold-induced lymphocyte transformation suggests an immunologic mechanism, ${ }^{5}$ none has yet been reported in isolated neutropenia. ${ }^{6}$

Gold-induced depression of bone marrow colony formation may be dose dependent and reversible on withdrawal. ${ }^{2}$ It has been suggested that this is the result of either direct inhibition of a colony-stimulating factor or impaired release of a colony-stimulating factor. ${ }^{7}$ The chronology of the current case and positive serologic findings render the possibility of coexistent cyclic neutropenia or chronic hypoplastic neutropenia untenable. ${ }^{8}$ In addition, the possibility of increased neutrophil consumption from chronic infection or within the reticuloendothelial system is refuted by lack of increased numbers of bone marrow precursors.

The absence of splenomegaly, rheumatoid nodules, or deformities in this patient renders a diagnosis of Felty's syndrome unlikely; how- ever, Felty's syndrome is occasionally seen without splenomegaly. Although bone marrow abnormalities are frequently seen in patients with Felty's syndrome, ${ }^{9}$ these abnormalities are notoriously either maturation arrest or increased numbers of granulocyte precursors, neither of which was seen in this patient's bone marrow specimen.

Rosenthal and associates ${ }^{9}$ have suggested that in the vast majority of Felty's syndrome cases specific granulocyte antibodies can be detected and subsequently result in phagocytosis of the leukocytes within the spleen (associated with increased bone marrow precursors) or within the bone marrow (maturation arrest). Antibody-mediated leukopenia in Felty's syndrome is supported by the development of leukopenia in normal patients given a transfusion of plasma obtained from a patient with Felty's syndrome. ${ }^{10}$ In the series by Rosenthal and associates, ${ }^{9}$ the authors felt that, in the two cases where there was a reduction in bone marrow precursors, this was likely to be secondary to concurrent antirheumatic therapy 
(gold). It is conceivable that gold therapy could have stimulated a lymphocyte mitogen response in the current case, perhaps secondary to inhibition of T-suppressor function, which, in turn, released latent autoimmune activity directed at the bone marrow. ${ }^{11}$ However, one would have expected that this would result in either a maturation arrest or increased numbers of granulocyte precursors in the bone marrow. Indeed, this was not the case. Therefore, we feel it is most likely that gold therapy had a direct toxic effect on the bone marrow, resulting in decreased granulocyte precursors.

The relative freedom of this patient from serious life-threatening infections remains enigmatic, but is undoubtedly related to her ability to augment another phagocytic cell line. The remarkable phagocytic activity of the patient's monocytes appears to have well compensated for her neutropenia.

1. Kay AGL: Myelotoxicity of gold. $B r$ Med $J$ 1976;1:12661268.

2. Howell A, Gumpel JM, Watt RWE: Depression of bone marrow colony formation in gold-induced neutropenia. $\mathrm{Br} \mathrm{Med} J$ 1975;1:432-434.

3. Westwick WJ, Allsop J, Gumpel JM, et al: Studies on pyrimidine biosynthesis in the granulocytes of patients receiving gold therapy for rheumatoid arthritis. $Q J \mathrm{Med} 1974 ; 43: 231$ 243.

4. Pisciotta AV: Immune and toxic mechanisms in drug-induced agranulocytosis. Semin Hematol 1973;10:279-310.

5. Denman EJ, Denman AM: The lymphocyte transformation test and gold hypersensitivity. Ann Rheum Dis 1968;27:582586.

6. Thompson DM, Pegelew CH, Singsen BH, et al: Neutropenia associated with chrysotherapy for juvenile rheumatoid arthritis. J Pedatr 1978;93:871-875.

7. Howell A, Chinn S, Andrews TM, et al: The effects of drugs that cause neutropenia upon colony formation by bone marrow cells in semi-solid agar. Clin Sci Mol Med 1974;46:619-628.

8. Spaet TH, Damesheck W: Chronic hypoplastic neutropenia. Am J Med 1952;13:35-45.

9. Rosenthal FD, Beeley JM, Gelsthorpe K, et al: White cell antibodies and the etiology of Felty's syndrome. $Q \mathrm{~J} \mathrm{Med}$ 1974;43:187-203.

10. Calabresi P, Edwards EE, Schilling RF: Fluorescent antiglobulin studies in leukopenic and related disorders. $J$ Clin Invest 1959;38:2091-2100.

11. Godfrey NF, Peter A, Simon TM, et al: IVN-acetylcysteine treatment of hematological reactions to chrysotherapy. J Rheumatol 1982;9:519-526. 


\section{You can talk to your patients about drugs.}

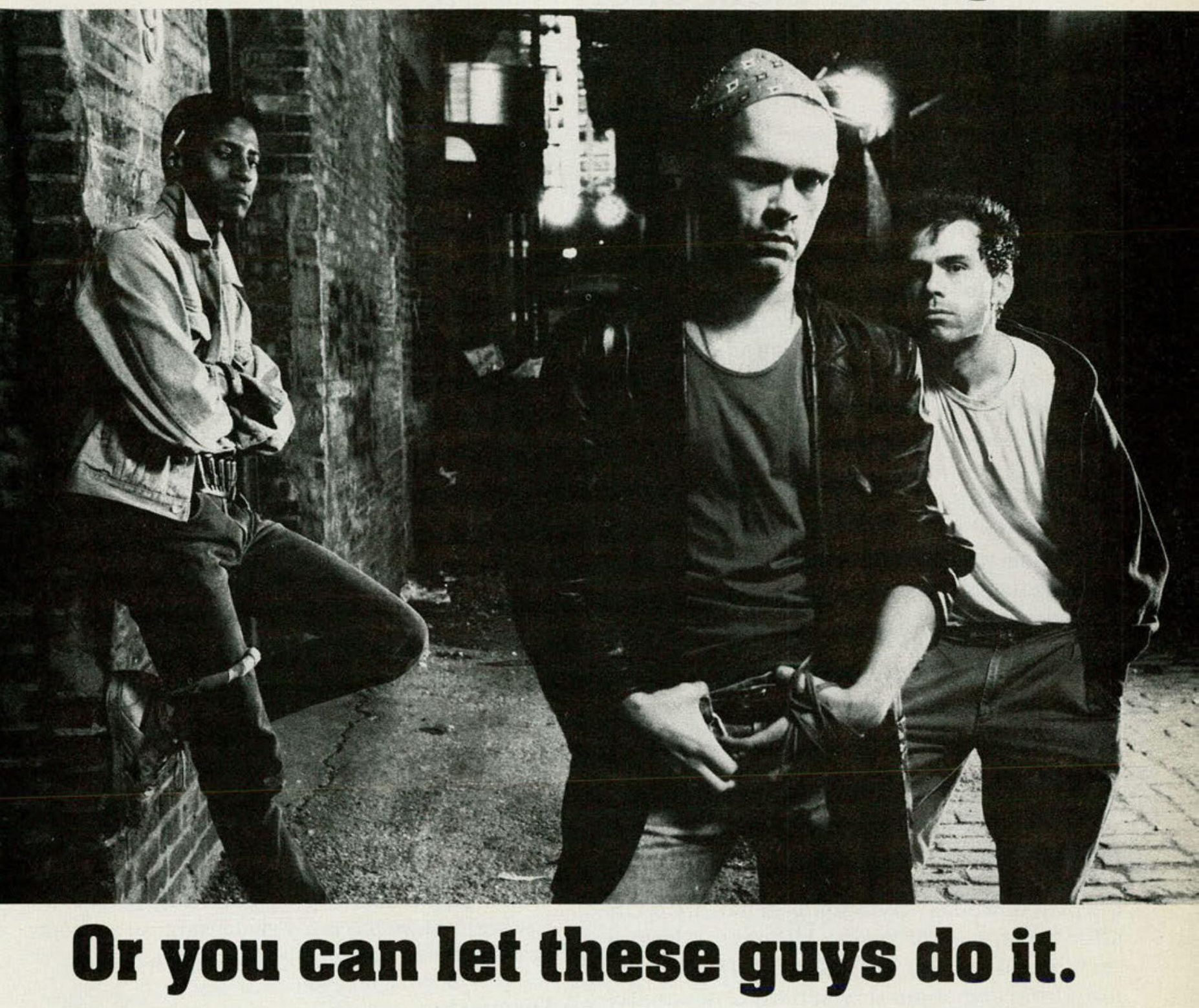

If you don't discuss illegal drugs with your patients, there are plenty of people who will. And some of them can be mighty persuasive.

That's why it's vital that you be heard. That you challenge the all-too-common acceptance of illegal drugs in American life. That you communicate to your patients the dangers of drug abuse. That you demonstrate you care.
Let's face it: yours is a powerful voice. A voice that can make a big difference to someone wrestling with the drug issue.

So if you have patients who you suspect may be abusing drugs or considering the use of illegal drugs, give them some straight talk.

Before these guys give them a sales talk.

\section{Start talking. Today.}

